\title{
Cytokine Signature in End-Stage Renal Disease Patients on Hemodialysis
}

\author{
Danyelle Romana Alves Rios, ${ }^{1}$ Melina Barros Pinheiro, ${ }^{1}$ Wander Valadares de Oliveira Junior, ${ }^{1}$ \\ Karina Braga Gomes, ${ }^{2}$ Andréa Teixeira Carvalho, ${ }^{3}$ Olindo Assis Martins-Filho, ${ }^{3}$ \\ Ana Cristina Simões e Silva, ${ }^{4}$ and Luci Maria Sant'Ana Dusse ${ }^{2}$ \\ ${ }^{1}$ Campus Centro Oeste, Federal University of Sao Joao del-Rei, Divinópolis, MG, Brazil \\ ${ }^{2}$ Department of Clinical and Toxicological Analysis, Faculty of Pharmacy, Federal University of Minas Gerais, Belo Horizonte, \\ MG, Brazil \\ ${ }^{3}$ Laboratório de Biomarcadores de Diagnóstico e Monitoração, Centro de Pesquisas René Rachou, Fundação Oswaldo Cruz, \\ Belo Horizonte, $M G$, Brazil \\ ${ }^{4}$ Department of Pediatrics, Interdisciplinary Laboratory of Medical Investigation, Faculty of Medicine, Federal University of Minas \\ Gerais, Belo Horizonte, MG, Brazil
}

Correspondence should be addressed to Danyelle Romana Alves Rios; danyelleromana@gmail.com

Received 3 May 2017; Revised 9 June 2017; Accepted 14 June 2017; Published 27 July 2017

Academic Editor: Shih-Ping Hsu

Copyright (c) 2017 Danyelle Romana Alves Rios et al. This is an open access article distributed under the Creative Commons Attribution License, which permits unrestricted use, distribution, and reproduction in any medium, provided the original work is properly cited.

\begin{abstract}
Hemodialysis is a modality of blood filtration in which accumulated toxins and water are removed from the body. This treatment is indicated for patients at the end stage of renal disease. Vascular access complications are responsible for 20-25\% of all hospitalizations in dialyzed patients. The occurrence of thrombosis in the vascular access is a serious problem that may severely compromise or even make the hemodialysis impossible, which is vital for the patient. The aim of this study was to investigate inflammatory profile in patients undergoing hemodialysis as well as the association between these alterations and vascular access thrombosis. A total of 195 patients undergoing hemodialysis have been evaluated; of which, 149 patients had not experienced vascular access thrombosis (group I) and 46 patients had previously presented this complication (group II). Plasma levels of cytokines including interleukin (IL-) 2 , IL-4, IL-5, IL-10, TNF- $\alpha$, and IFN- $\gamma$ were measured by cytometric bead array. Our results showed that patients with previous thrombotic events (group II) had higher levels of the IL-2, IL-4, IL-5, and IFN- $\gamma$ when compared to those in group I. Furthermore, a different cytokine signature was detected in dialyzed patients according to previous occurrences or not of thrombotic events, suggesting that elevated levels of T-helper 1 and T-helper 2 cytokines might, at least in part, contribute to this complication.
\end{abstract}

\section{Introduction}

Hemodialysis (HD) requires a well-functioning vascular access that allows sufficient blood flow to achieve adequate clearance and blood dialysis. Vascular access complications increase morbidity and are responsible for 20-25\% of all hospitalizations in dialyzed patients; of which, approximately $85 \%$ of the cases are due to thrombosis $[1,2]$. Although substantial progress has been achieved in preventing thrombotic complications in various groups of patients, the mechanisms of thrombosis during hemodialysis still require clarification.

End-stage renal disease (ESRD) and HD itself lead to an inflammatory status induced by multiple factors, including uremic syndrome per se, heart failure, vascular access infections, bioincompatible dialysis solutions, the accumulation of advanced glycation products, progressive decrease of glomerular filtration rate [3-5], and blood-membrane interaction [6]. Chronic inflammation as a major determinant of 
"dialysis syndrome" is considered as the main factor of morbidity and mortality in dialysis patients. Therefore, the immune system is recognized as one of the most important therapeutic targets to reduce morbidity and mortality in uremic and dialysis patients [7].

Inflammation is characterized by the enhanced production of C-reactive protein (CRP) and other inflammatory mediators, including T-helper 1 (Th-1) cytokines such as tumor necrosis factor- $\alpha$ (TNF- $\alpha$ ), interleukin (IL-) 6 , and IL-2 as well chemokines such as IL-8 [8]. Although viewed separately, coagulation and inflammation are highly related. Both biological processes interact and cooperate for the organism response to injury and invasion by pathogens. It is known that the coagulation proteins, besides acting in cascade promoting the formation of the fibrin clot, also have an isolated action in other pathophysiological processes. In this way, the tissue factor (TF), factor VII, factor X, and thrombin have an important role in inflammatory process [9]. It is also known that CRP, TNF- $\alpha$, and IL- 1 stimulate TF expression by monocytes and endothelial cells $[10,11]$. Dysregulation of any component of these systems can affect the entire balance, resulting in a wide range of illnesses that may result in variable degrees of inflammation and thrombosis [12].

Inflammation in dialysis patients may vary over time and during this process. Anti-inflammatory and modulatory cytokines can also be produced as an attempt to control this process, such as IL-4, IL-5, and IL-10. IL-10 is secreted by lymphocytes of the T-helper 2 (Th2) subtype and also in large amounts by macrophages [13]. IL-10 is an antiinflammatory cytokine with potent deactivating properties on both macrophages and T cells. IL-10 inhibits several cellular processes responsible for plaque progression, rupture, or thrombosis, including nuclear factor $-\kappa \mathrm{B}(\mathrm{NF}-\kappa \mathrm{B})$ activation, metalloproteinase production, $\mathrm{TF}$ and cyclooxygenase-2 expression, and cell death $[13,14]$. It is known that IL-4 can modulate the already existing inflammatory process and can also induce the expression of t-PA that contributes to the activation of plasminogen in the patients who presented thrombotic events [15].

The aim of this study was to evaluate plasma levels of Th- 1 and Th-2 cytokines in patients undergoing hemodialysis and the association of the cytokine profile with thrombotic complications in vascular access.

\section{Methods}

2.1. Study Design. This was an observational cross-sectional study.

2.2. Patients. The present study included HD patients for more than six months $(n=195)$ with arteriovenous fistula, aged between 18 and 70 years, selected from two dialysis centers in Belo Horizonte, MG, Brazil, according to the exclusion and inclusion criteria.

The HD patients were allocated into two subgroups, according to the occurrence or not of a previous episode of vascular access thrombosis (VAT) (with and without VAT). HD patients with VAT (group II) consisted of 46 individuals whose functioning dialysis access had, at least, one previous episode of clinically evident thrombotic occlusion. Thrombotic occlusion was defined by the absence of blood flow and the impossibility to use the access for dialysis. The remained HD patients consisted of 149 subjects who have not experienced this complication (group I).

All patients required regular HD sessions for 3 to 4 hours, three times a week. Blood flow was usually $300-450 \mathrm{~mL} / \mathrm{min}$ with a dialysate flow at a constant rate of $500 \mathrm{~mL} / \mathrm{min}$. Patients were dialyzed either with low-flux polysulphone membranes or high-flow polysulphone membranes with bicarbonate-buffered dialysate. All patients received regular doses of standard heparin (100 to $150 \mathrm{UI} / \mathrm{kg}$ ) before a hemodialysis session.

Exclusion criteria were hemodialysis patients receiving oral anticoagulation therapy or oral contraceptives, with prior history of arterial or venous thrombosis (except those with VAT), with acute or chronic hepatic disease, autoimmune and malignant diseases, vasculitis, acute infections, clinical instabilities, a history of renal transplantation, and HIV-positive and pregnant women.

2.3. Ethical Considerations. The ethics committee of our institution approved the study, and informed consent was obtained from all participants. The research protocol did not interfere with any medical recommendations or prescriptions.

2.4. Variables. A detailed history, clinical variables [age, gender, body mass index (BMI), predialysis blood pressure levels, etiology of ESRD, presence of diabetes or not, type of vascular access, time on hemodialysis, and interdialytic weight gain], and laboratorial parameters including known factors affecting thrombosis such as platelet numbers, hsCRP, leukocytes, and erythrocytes, as well as nutritional parameters such as albumin and normalized protein catabolic rate (nPCR) of each included patient were recorded retrospectively in a computer-specific data bank.

After informed consent, all subjects were submitted to blood collection for the determination of plasma levels of cytokines.

2.5. Cytometric Bead Array for Cytokine Measurements. Blood samples were drawn in EDTA-K $\mathrm{K}_{3} \quad 1.8 \mathrm{mg} / \mathrm{mL}$ (Vacuette ${ }^{\circledR}$ ), using a $16 \mathrm{G}$ needle, from HD vascular access prior to dialysis procedure at the first dialysis session of the week and before heparin administration. Samples were centrifuged at $2500 \mathrm{~g}$ for $20 \mathrm{~min}$ at $4^{\circ} \mathrm{C}$ to obtain plasma, which was stored at $-70^{\circ} \mathrm{C}$ until analysis.

Cytokine plasma levels acquisition and analysis were performed in a dual-laser FACSCalibur ${ }^{\mathrm{TM}}$ flow cytometer (BD Biosciences Pharmingen, San Jose, CA, USA) using the BD Bioscience CBA software. IL-2, IL-4, IL-5, IL-10, IFN- $\gamma$, and TNF- $\alpha$ were determined using the Human Th1/Th2 cytometric bead array method (BD Biosciences Pharmingen, USA), as previously described by Pinheiro et al. [16].

2.6. Analysis of Plasma Levels of Cytokines and Cytokine Signature. Plasma levels of cytokines were analyzed as the mean fluorescence intensity (MFI) provided by the CBA immunoassay. Additionally, the analysis referred as "cytokine signature" was also performed as previously proposed 
TABle 1: Clinical characteristics and dialysis parameters of hemodialysis patients with (cases) and without (controls) previous episode of vascular access thrombosis.

\begin{tabular}{|c|c|c|c|}
\hline Parameters & Group I $(n=149)$ & Group II $(n=46)$ & $p$ value \\
\hline Age (years) & $52(39-60)$ & $50(41-59)$ & 0.380 \\
\hline Gender & & & 0.193 \\
\hline Male $[n(\%)]$ & $86(58 \%)$ & $20(43 \%)$ & \\
\hline Female $[n(\%)]$ & $63(42 \%)$ & $26(57 \%)$ & \\
\hline BMI $\left(\mathrm{kg} / \mathrm{m}^{2}\right)$ & $23.6(21.1-26.5)$ & $22.6(20.1-28.5)$ & 0.112 \\
\hline \multicolumn{4}{|l|}{ Cause of ESRD [ $n(\%)]$} \\
\hline Hypertensive nephrosclerosis & $51(34 \%)$ & $14(30 \%)$ & 0.633 \\
\hline Glomerulopathies & $39(26 \%)$ & $7(15 \%)$ & 0.126 \\
\hline Diabetic nephropathy & $21(14 \%)$ & $12(26 \%)$ & 0.060 \\
\hline Polycystic kidney disease & $7(5 \%)$ & $3(7 \%)$ & 0.624 \\
\hline Others or unknowns causes & $31(21 \%)$ & $10(22 \%)$ & 0.892 \\
\hline \multicolumn{4}{|l|}{ Predialysis arterial blood pressure } \\
\hline Systolic blood pressure (mmHg) & $130(120-143)$ & $140(130-150)$ & 0.060 \\
\hline Diastolic blood pressure ( $\mathrm{mmHg}$ ) & $80(80-90)$ & $80(80-90)$ & 0.968 \\
\hline Type of vascular access & & & 0.131 \\
\hline Arteriovenous fistula & $144(97 \%)$ & $42(91 \%)$ & \\
\hline Arteriovenous graft & $5(3 \%)$ & $4(9 \%)$ & \\
\hline Time on hemodialysis (months) & $34.0(17.0-90.3)$ & $39.5(19.0-92.0)$ & 0.226 \\
\hline IWG $(\mathrm{kg})$ & $3.2 \pm 1.1$ & $2.9 \pm 1.4$ & 0.165 \\
\hline \multicolumn{4}{|l|}{ Medications $[n(\%)]$} \\
\hline \multicolumn{4}{|l|}{ Antihypertensive drugs } \\
\hline ACE inhibitor & $69(46 \%)$ & $69(46 \%)$ & 0.551 \\
\hline$\beta$-Blockers & $64(43 \%)$ & $21(46 \%)$ & 0.747 \\
\hline Calcium channel antagonists & $65(44 \%)$ & $17(37 \%)$ & 0.423 \\
\hline Acetylsalicylic acid & $38(26 \%)$ & $8(17 \%)$ & 0.257 \\
\hline Statins & $31(21 \%)$ & $5(11 \%)$ & 0.129 \\
\hline Insulin & $29(19 \%)$ & $10(22 \%)$ & 0.736 \\
\hline Erythropoietin & $129(87 \%)$ & $41(89 \%)$ & 0.651 \\
\hline Diabetes $[n(\%)]$ & $40(27 \%)$ & $15(33 \%)$ & 0.448 \\
\hline
\end{tabular}

The normally distributed data were expressed as mean \pm SD ( $t$-test). The non-Gaussian data were presented as median (range) (Mann-Whitney $U$ test). Frequencies (\%) were evaluated by $\chi 2$ test. Group I: patients without VAT. Group II: patients with VAT. BMI: body mass index; ESRD: end stage of renal disease; IWG: interdialytic weight gain.

by Luiza-Silva et al. and Pinheiro et al. [16, 17]. Briefly, the global median value for each cytokine was calculated taking the whole data universe from all patients. The global median cut-off was used as the cut-off edge to tag each patient as they display "low levels" ( $\square$ for all cytokines) and "high levels" ( for all cytokines). The frequency (\%) of patients showing "high cytokine levels" was calculated, and the "cytokine signature" assembled as the ascendant frequencies in order to identify changes in the overall cytokine patterns between the two studied groups.

2.7. Statistical Analysis. Variables were checked for Gaussian distribution by the Shapiro-Wilk test. Non-Gaussian data were expressed as median and interquartile range (25th percentile and 75th percentile). Demographic, clinical characteristic and plasma levels of cytokines, expressed as MFI, were compared among groups by Mann-Whitney $U$ test. All analyses were performed using the program GraphPad
PRISM (version 5.0), and significant differences, in all cases, are considered at $p<0.05$. Additional strategy of data analysis was used to compare the ascendant profile referred as "cytokine signature." Relevant differences were considered when the frequency of patients with "high cytokine levels" was above the 50th percentile.

\section{Results}

Clinical variables, laboratorial and nutritional parameters, did not differ between group I and group II, as displayed in Tables 1 and 2. Age, gender, body mass index (BMI), predialysis blood pressure levels, etiology of ESRD, presence of diabetes or not, type of vascular access, time on hemodialysis, and interdialytic weight gain were similar in both groups ( $p>0.05$ for all comparisons).

To characterize the overall cytokine pattern of hemodialysis patients with or without VAT, we have first compared 
TABLE 2: Laboratorial parameters of hemodialysis patients with (cases) and without (controls) previous episode of vascular access thrombosis.

\begin{tabular}{|c|c|c|c|}
\hline Parameters & Group I $(n=149)$ & Group II $(n=46)$ & $p$ value \\
\hline Erythrocytes $\times 10^{6} / \mathrm{mL}$ & $4.1(3.6-4.5)$ & $3.9(3.6-4.2)$ & 0.143 \\
\hline Hemoglobin (g/dL) & $12.4(11.1-13.8)$ & $11.9(10.7-13.1)$ & 0.107 \\
\hline Hematocrit (\%) & $36.7 \pm 5.7$ & $35.7 \pm 5.1$ & 0.288 \\
\hline $\mathrm{MCV}$ & $91.3 \pm 5.6$ & $91.3 \pm 5.0$ & 0.956 \\
\hline $\mathrm{MCH}$ & $30.3 \pm 2.2$ & $30.0 \pm 2.1$ & 0.405 \\
\hline MCHC & $33.3(32.8-33.6)$ & $33.2(32.4-33.7)$ & 0.440 \\
\hline Platelets $\times 10^{3} / \mathrm{mL}$ & $210.0(175.0-264.0)$ & $224.5(176.0-254.0)$ & 0.693 \\
\hline Leukocytes $\times 10^{3} / \mathrm{mL}$ & $7.0(5.0-8.0)$ & $6.2(5.7-7.5)$ & 0.685 \\
\hline Serum iron $(\mu \mathrm{g} / \mathrm{dL})$ & $58.0(45.8-74.0)$ & $54.0(42.8-70.8)$ & 0.443 \\
\hline TIBC $(\mu \mathrm{g} / \mathrm{dL})$ & $229.0 \pm 48.0$ & $226.0 \pm 45.0$ & 0.774 \\
\hline TS (\%) & $25.3(19.0-34.0)$ & $24.1(20.0-31.0)$ & 0.587 \\
\hline Ferritin (ng/mL) & $340.0(208.7-610.3)$ & $319.5(154.0-556.0)$ & 0.198 \\
\hline TC (mg/dL) & $164.3 \pm 38.7$ & $166.3 \pm 34.5$ & 0.747 \\
\hline $\mathrm{LDLc}(\mathrm{mg} / \mathrm{dL})$ & $93.2 \pm 30.0$ & $97.1 \pm 25.0$ & 0.440 \\
\hline $\mathrm{HDLc}(\mathrm{mg} / \mathrm{dL})$ & $34.0(29.0-44.0)$ & $33.0(27.0-43.0)$ & 0.304 \\
\hline TG (mg/dL) & $150.0(90.5-229.5)$ & $126.0(100.0-234.0)$ & 0.983 \\
\hline hsCRP & $3.7(1.7-8.4)$ & $3.2(1.6-8.9)$ & 0.811 \\
\hline Total protein $(\mathrm{g} / \mathrm{dL})$ & $7.5 \pm 0.7$ & $7.3 \pm 0.8$ & 0.095 \\
\hline Creatinin (mg/dL) & $12.0 \pm 3.4$ & $11.4 \pm 3.0$ & 0.322 \\
\hline Albumin (g/dL) & $3.5(3.3-3.8)$ & $3.6(3.4-3.8)$ & 0.290 \\
\hline nPCR (g/kg/dia) & $1.1(1.0-1.4)$ & $1.2(1.0-1.6)$ & 0.451 \\
\hline
\end{tabular}

The normally distributed data were expressed as mean \pm SD ( $t$-test). The non-Gaussian data were presented as median (range) (Mann-Whitney test). MCV: mean corpuscular volume; MCH: mean corpuscular hemoglobin; MCHC: mean corpuscular hemoglobin concentration; TIBC: total iron binding capacity; TS: transferrin saturation; TC: total cholesterol; LDLc: low-density lipoprotein cholesterol; HDLc: high-density lipoprotein cholesterol; TG: triglycerides; hsCRP: high sensitivity C-protein reactive; nPCR: normalized protein catabolic rate.

plasma levels of cytokines according to the presence (group II) or absence of thrombotic events (group I). In these comparisons, no statistical difference was observed (Figure 1). We also did the comparison among groups regarding the ratio of pro- (IL-2, TNF- $\alpha$, and IFN- $\gamma$ ) versus antiinflammatory cytokine (IL-10), and no difference was found (data not shown).

In order to assemble the "cytokine signature" of each study group, the global median value was determined for each cytokine: IL- $2=1.3$, IL- $4=2.4$, IL-5 = 5.0, IL-10 = 6.6, TNF- $\alpha=4.7$, and IFN- $\gamma=5.6$, all expressed in MFI. These values were used to establish the cut-off point used to separate patients with "low" or "high" cytokine levels. Using these criteria, each one received a tag for each cytokine. Following, diagrams were used to assemble the proinflammatory and anti-inflammatory profiles and to calculate the frequency (\%) of patients showing "high cytokine levels" as showed in Figure 2.

The frequency of patients with high cytokine levels was further compiled to establish the cytokine-ascendant profile, referred as "cytokine signature" for each study group (Figure 3(a)). Data analysis was carried out considering only the relevant cytokine frequencies above the 50th percentile. According to this approach, in patients who had a previous episode of VAT, the relevant components of the "cytokine signature" are the inflammatory cytokines, IL-2 and IFN- $\gamma$, and the modulatory Th- 2 cytokines, IL- 4 and IL-5. On the other hand, patients who have not experienced previous episode of VAT showed an increased frequency of only antiinflammatory and modulatory cytokines, IL-10 and IL-4, respectively. The overlay of ascendant "cytokine signature" from the two study groups was further used to illustrate these findings (Figure 3(b)).

For better understanding of the results, the ascendant "cytokine signature" from patients who had no previous episode of VAT (group I) was used as a reference curve for comparative analysis with the patients who had a previous episode of VAT (group II) (Figure 4). Considering only the relevant cytokine frequencies above the 50th percentile, data analysis showed higher frequency of inflammatory cytokines, IL-2 and IFN- $\gamma$, and only one modulatory cytokine, IL-5, along with lower frequency of the anti-inflammatory cytokine, IL-10 (Figure 4).

\section{Discussion}

In the present study, the plasma levels of Th- 1 and Th- 2 cytokines were evaluated and a comparison was made between patients who previously presented VAT and those who did not. Indeed, no significant differences were detected in the comparison of both groups of ESRD patients on hemodialysis. It is important to highlight that, due to the cross-sectional design of our study, the evaluations were performed only in a single time point for all patients. Therefore, it is not possible 


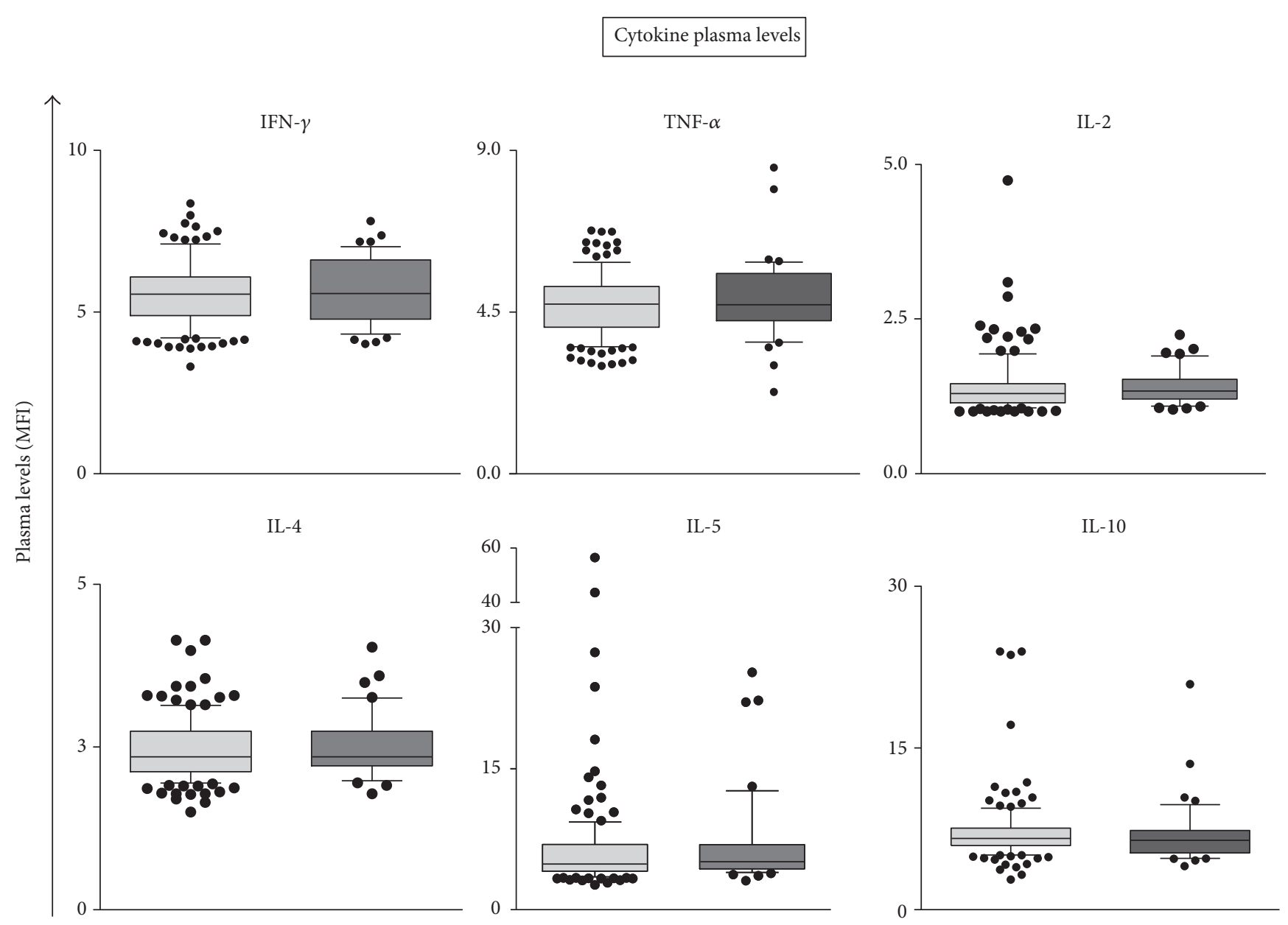

Figure 1: Cytokine plasma levels in group I ( $\square$ ) as compared to those in group II ( $\square$ ). Plasma levels of proinflammatory (IL-2, TNF- $\alpha$, and IFN- $\gamma$ ) and regulatory (IL-4, IL-5, and IL-10) cytokines were determined by cytometric bead array. Results are expressed in mean fluorescence intensity (MFI); data are presented in a box-plot format. The median is shown as a line across the box. Group I: HD patients without VAT. Group II: HD patients with episodes of VAT.
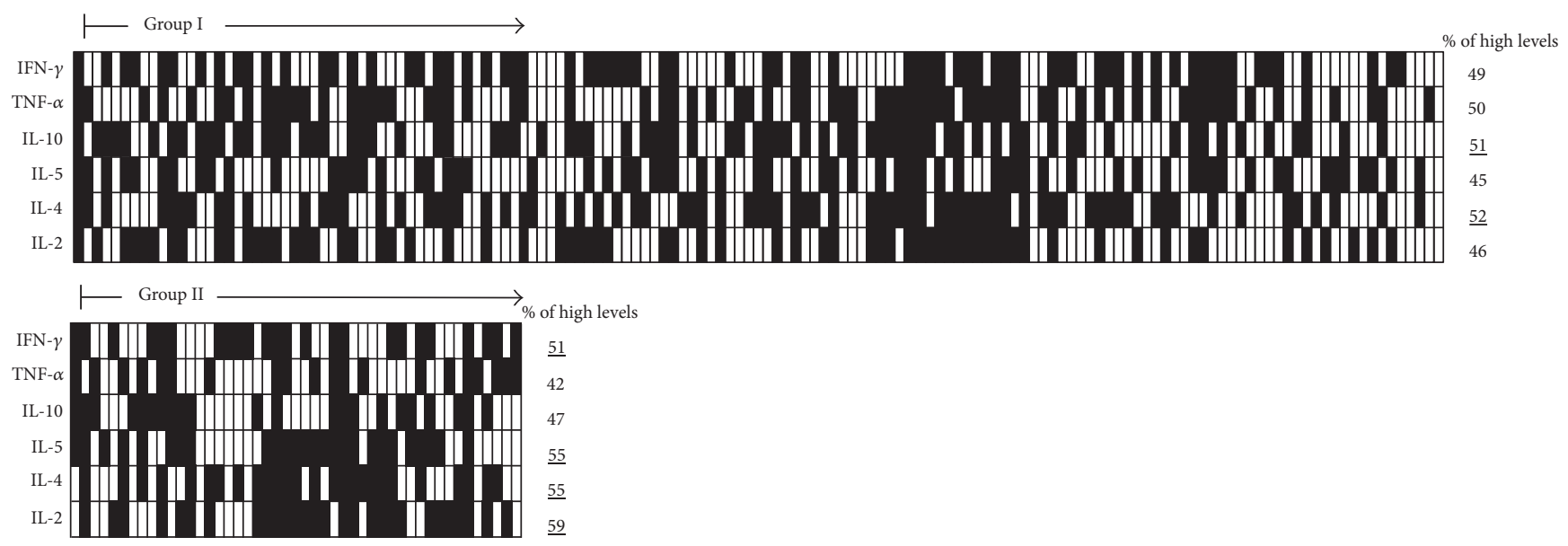

FIGURE 2: Multicytokine diagrams were used to quantify the frequency of HD patients with high levels of cytokines in the studied group. The global median value for each cytokine was used as the cut-off edge to tag each patient as they display "low levels" ( $\square$ for all cytokines) and "high levels" of proinflammatory or regulatory ( $\square$ for IL-2, IL-4, IL-5, IL-10, TNF- $\alpha$, and IFN- $\gamma$ ) cytokines. Group I: HD patients without VAT. Group II: HD patients with episodes of VAT. 
Group I

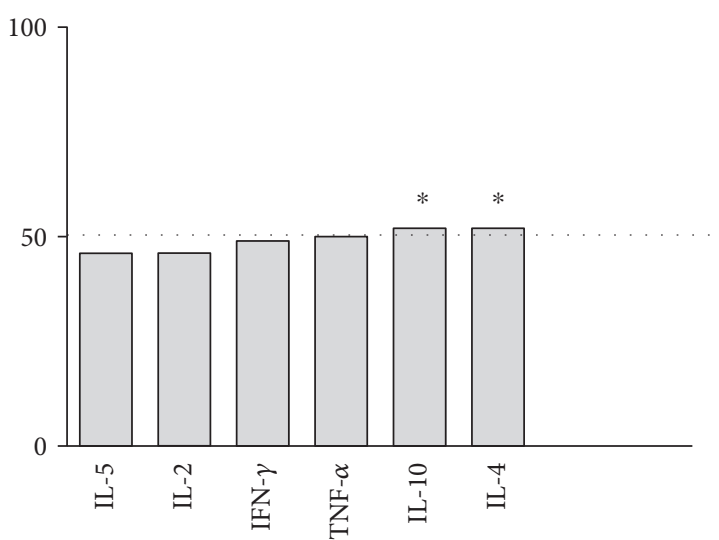

(a)

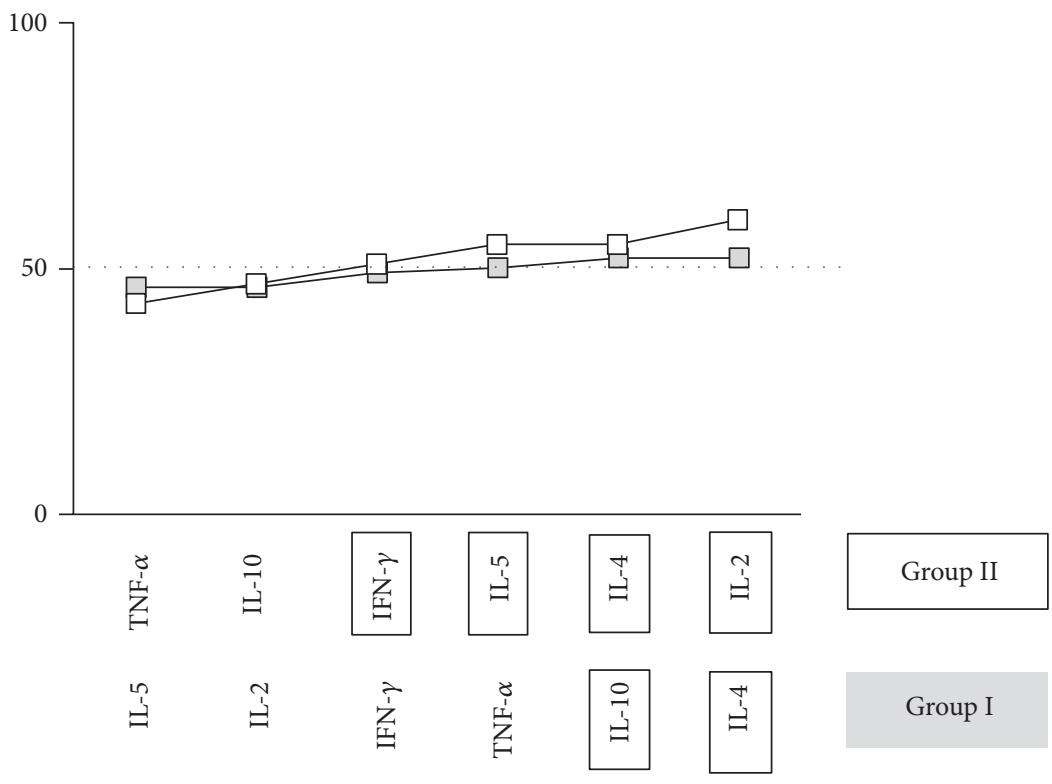

(b)

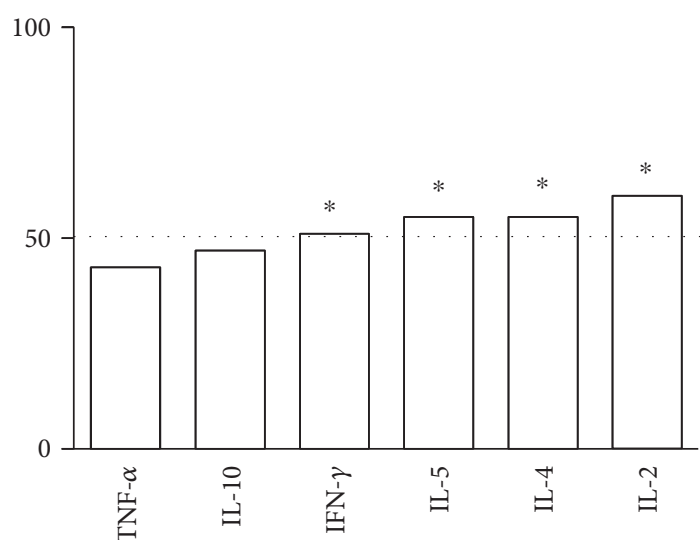




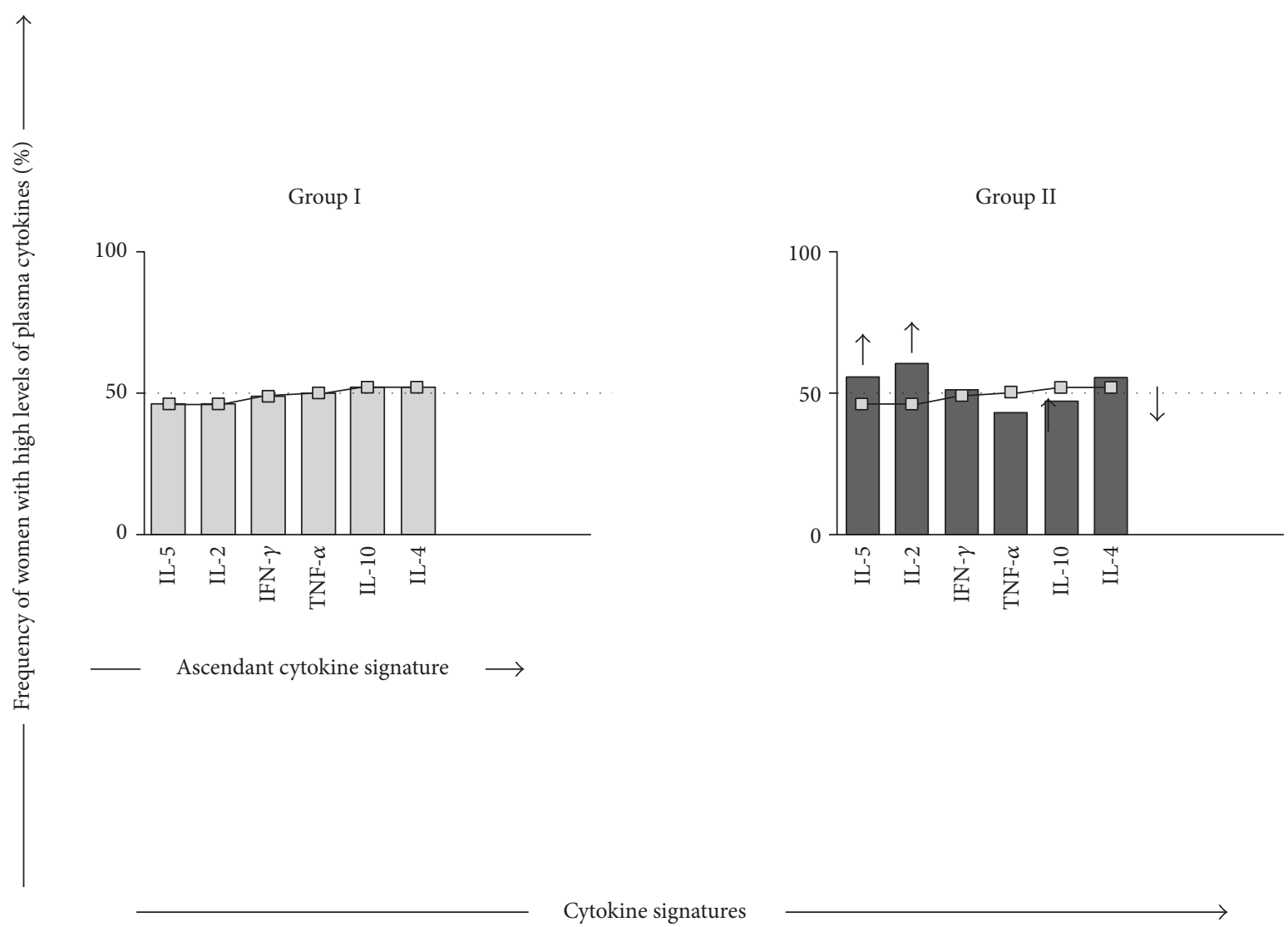

FIGURE 4: Comparative analysis of the cytokine signatures between group I ( $\square$ ) and group II ( $\square$ ). The ascendant frequency of group I with high cytokine plasma levels was assembled for group II as demonstrated by bar graphs and by the ascendant cytokine curve for group I (- - ). The ascendant frequency of group II with high cytokine plasma levels was also assembled for group I (- - ) and used for comparative analysis with the cytokine profile of HD patients with VAT. Dotted lines indicate the 50th percentiles used as the cut-off to identify relevant elements, highlighted by $\uparrow$, for increased frequencies. Group I: HD patients without VAT. Group II: HD patients with VAT.

"cytokine signature" from the patients who have not experienced VAT was compared with patients that previously had VAT (Figure 4). In this way, only the cytokine frequencies above the 50th percentile were considered. The ascendant "cytokine signature" analysis revealed that the VAT group displayed an elevated percentage of patients with high levels of IL-2, IL-5, and IFN- $\gamma$ and lower frequency of the antiinflammatory cytokine, IL-10. These data suggest that thrombotic events may be associated with an unbalance between proinflammatory and anti-inflammatory cytokines.

Once thrombotic events in coronary arteries and deep limbs are associated with inflammation of the affected vessels, it can be considered that, in VAT, inflammation is involved in the thrombosis process [9-11]. However, the association between the presence of inflammation and complications of vascular access is still not very well established. In this regard, Liu et al. [18] observed that high-sensitivity C-reactive protein (hsCRP), TNF- $\alpha$ and IL-6 levels were significantly higher in hemodialysis patients who developed VAT $(n=14)$ when compared to the group of patients who did not have this complication $(n=18)$ and to the group of patients initiating hemodialysis $(n=15)$. The authors also observed that patients with VAT presented significant hyperplasia of the intimal layer of the vascular wall of the VAT and increased monocyte chemotactic protein (MCP-1) expression compared to those in the group that was initiating the treatment, and these alterations were positively correlated to increased levels of hsCRP. The authors hypothesized that microinflammation may be involved in the development of intimal hyperplasia, leading to stenosis and, consequently, VAT [19].

In response to vascular injury, smooth muscle cells undergo phenotypic changes characterized by increased affinity of integrins for their ligands, expression of adhesion molecules, cytokines, and growth factors, including IL-1, MCP-1, transforming growth factor beta 1 (TGF- $\beta 1$ ), and TNF- $\alpha[18,19]$. These alterations facilitate leukocyte chemotaxis and vessel wall infiltration, as well as stimulate the expression of adhesion molecules on the endothelium and the production of extracellular matrix components, including collagen, elastin, and proteoglycans [20].

Chang et al. [21] observed abundant and moderate infiltration of macrophages and lymphocytes into the vascular appearance of VATs that developed thrombosis $(n=23)$ accompanied by increased expression of vascular adhesion molecule-1 (VCAM-1), proinflammatory cytokines (IL-6 and TNF- $\alpha$ ), and matrix-9 metalloproteinase (MMP-9) compared to the vascular wall of vascular access that did not 
develop thrombosis $(n=13)$. The authors proposed that IL-6 and VCAM-1 might play a role in increasing proliferation of vascular smooth muscle cells and MMP-9, thus causing instability in the neointimal layer and subsequent injury of the endothelium, leading to thrombosis. They also suggested that anti-inflammatory treatment could be useful to improve the longevity of the vascular access.

Kourtzelis et al. [22] showed that complement activation triggered by hemodialysis biomaterials, and the subsequent generation of the complement anaphylatoxin $\mathrm{C} 5 \mathrm{a}$, resulted in the expression of functionally active TF in peripheral blood neutrophils [23]. Moreover, the persistent complement activation that occurs during long-term hemodialysis contributed to thrombosis in ESRD patients. The authors considered that complement contributed to the induction of granulocyte colony-stimulating factor, which has been implicated in the pathogenesis of thrombosis in patients treated with the recombinant form of this molecule, suggesting that the complement system could become a new therapeutic target for preventing thrombosis in patients on hemodialysis [23].

In summary, our data showed that the presence of thrombotic events in patients undergoing hemodialysis is associated with an elevated percentage of patients with high levels of proinflammatory cytokines (IL-2 and IFN- $\gamma$ ) and low levels of the anti-inflammatory cytokine (IL-10) that might drive the cytokine network in VAT patients towards an inflammatory state.

A study limitation of this study was that the time of vascular thrombosis occurrence and cytokines dosing was not to evaluat, and therefore, our findings only reveal associations and not causation due to the observational nature of the study. It should be pointed that longitudinal studies are needed to characterize the plasma cytokine profile in patients undergoing hemodialysis and its association with the occurrence of clinical complications, including thrombotic events. In addition, prospective studies might support the applicability of these biomarkers as prognostic tools for the follow-up of patients on hemodialysis.

\section{Conflicts of Interest}

The authors declare that there is no conflict of interest regarding the publication of this paper.

\section{Acknowledgments}

The authors thank FAPEMIG and CNPq/Brazil. Luci Maria Sant'Ana Dusse, Ana Cristina Simões e Silva, Olindo Assis Martins-Filho, and Andréa Teixeira Carvalho are grateful to $\mathrm{CNPq}$ Research Fellowship (PQ).

\section{References}

[1] H. I. Feldman, S. Kobrin, and A. Wasserstein, "Hemodialysis vascular access morbidity," Journal of the American Society of Nephrology, vol. 7, no. 4, pp. 523-535, 1996.

[2] J. H. Smits, J. van der Linden, P. J. Blankestijn, and T. J. Rabelink, "Coagulation and haemodialysis access thrombosis,"
Nephrology, Dialysis, Transplantation, vol. 15, no. 11, pp. 1755-1760, 2000.

[3] H. R. Vianna, C. M. B. M. Soares, M. S. Tavares, M. M. Teixeira, and A.C.S. Silva, "Inflamação na doença renal crônica: papel de citocinas," Jornal Brasileiro de Nefrologia, vol. 33, no. 3, pp. 351-364, 2011.

[4] A. Bruchfeld, J. J. Carrero, A. R. Qureshi et al., "Elevated serum macrophage migration inhibitory factor (MIF) concentrations in chronic kidney disease (CKD) are associated with markers of oxidative stress and endothelial activation," Molecular Medicine, vol. 15, no. 3-4, pp. 70-75, 2009.

[5] E. J. Sharples, M. Varagunam, P. J. Sinnott, D. J. McCloskey, M. J. Raftery, and M. M. Yaqoob, "The effect of proinflammatory cytokine gene and angiotensin-converting enzyme polymorphisms on erythropoietin requirements in patients on continuous ambulatory peritoneal dialysis," Peritoneal Dialysis International, vol. 26, no. 1, pp. 64-68, 2006.

[6] G. A. Kaysen, "The microinflammatory state in uremia: causes and potential consequences," Journal of the American Society of Nephrology, vol. 12, no. 7, pp. 1549-1557, 2001.

[7] M. R. Sharif, Z. Chitsazian, M. Moosavian et al., "Immune disorders in hemodialysis patients," Iranian Journal of Kidney Diseases, vol. 9, no. 2, pp. 84-96, 2015.

[8] S. M. d. Lima, A. Otoni, P. Sabino Ade et al., "Inflammation, neoangiogenesis and fibrosis in peritoneal dialysis," Clinica Chimica Acta, vol. 421, pp. 46-50, 2013.

[9] A. P. Mota, S. S. Vilaça, F. L. das Mercês et al., "Cytokines signatures in short and long-term stable renal transplanted patients," Cytokine, vol. 62, no. 2, pp. 302-309, 2013.

[10] P. Libby, "Molecular bases of the acute coronary syndromes," Circulation, vol. 91, no. 11, pp. 2844-2850, 1995.

[11] R. T. Lee and P. Libby, “The unstable atheroma," Arteriosclerosis, Thrombosis, and Vascular Biology, vol. 17, no. 10, pp. 1859-1867, 1997.

[12] J. H. Foley and E. M. Conway, "Cross talk pathways between coagulation and inflammation," Circulation Research, vol. 118, no. 9, pp. 1392-1408, 2016.

[13] X. Han and W. A. Boisvert, "Interleukin-10 protects against atherosclerosis by modulating multiple atherogenic macrophage function," Thrombosis and Haemostasis, vol. 113, no. 3, pp. 505-512, 2015.

[14] Z. Mallat, S. Besnard, M. Duriez et al., "Protective role of interleukin-10 in atherosclerosis," Circulation Research, vol. 85, no. 8, pp. e17-e24, 1999.

[15] P. H. Hart, D. R. Burgess, G. F. Vitti, and J. A. Hamilton, "Interleukin-4 stimulates human monocytes to produce tissue-type plasminogen activator," Blood, vol. 74 , no. 4, pp. 1222-1225, 1989.

[16] M. B. Pinheiro, O. A. Martins-Filho, A. P. Mota et al., "Severe preeclampsia goes along with a cytokine network disturbance towards a systemic inflammatory state," Cytokine, vol. 62, no. 1, pp. 165-173, 2013.

[17] M. Luiza-Silva, A. C. Campi-Azevedo, M. A. Batista et al., "Cytokine signatures of innate and adaptive immunity in 17DD yellow fever vaccinated children and its association with the level of neutralizing antibody," The Journal of Infectious Diseases, vol. 204, no. 6, pp. 873-883, 2011.

[18] B. C. Liu, L. Li, M. Gao, Y. L. Wang, and J. R. Yu, "Microinflammation is involved in the dysfunction of arteriovenous fistula in patients with maintenance hemodialysis," Chinese Medical Journal, vol. 121, no. 21, pp. 2157-2161, 2008. 
[19] T. W. Wakefield, R. M. Strieter, C. A. Wilke et al., "Venous thrombosis-associated inflammation and attenuation with neutralizing antibodies to cytokines and adhesion molecules," Arteriosclerosis, Thrombosis, and Vascular Biology, vol. 15, no. 2, pp. 258-268, 1995.

[20] A. Y. Tiong and D. Brieger, "Inflammation and coronary artery disease," American Heart Journal, vol. 150, no. 1, pp. 11-18, 2005.

[21] C. J. Chang, Y. S. Ko, P. J. Ko et al., “Thrombosed arteriovenous fistula for hemodialysis access is characterized by a marked inflammatory activity," Kidney International, vol. 68, no. 3, pp. 1312-1319, 2005.

[22] I. Kourtzelis, M. M. Markiewski, M. Doumas et al., "Complement anaphylatoxin $\mathrm{C} 5 \mathrm{a}$ contributes to hemodialysisassociated thrombosis," Blood, vol. 116, no. 4, pp. 631-639, 2010.

[23] V. N. Krishna, J. B. Eason, and M. Allon, "Central venous occlusion in the hemodialysis patient," American Journal of Kidney Diseases, vol. 68, no. 5, pp. 803-807, 2016. 


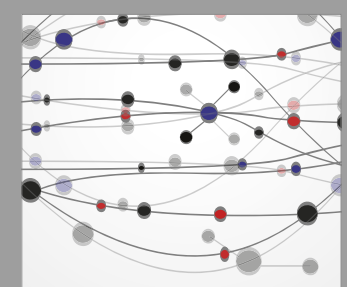

The Scientific World Journal
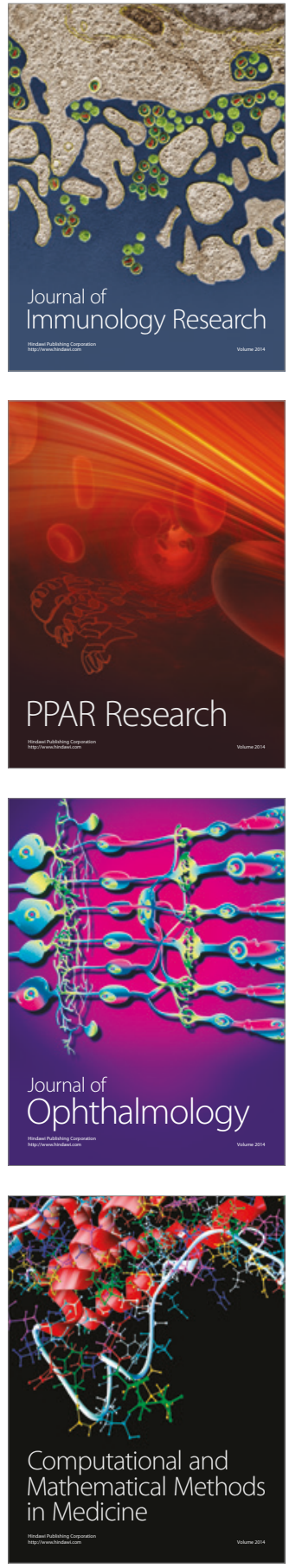

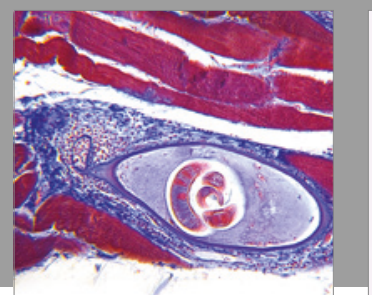

Gastroenterology Research and Practice
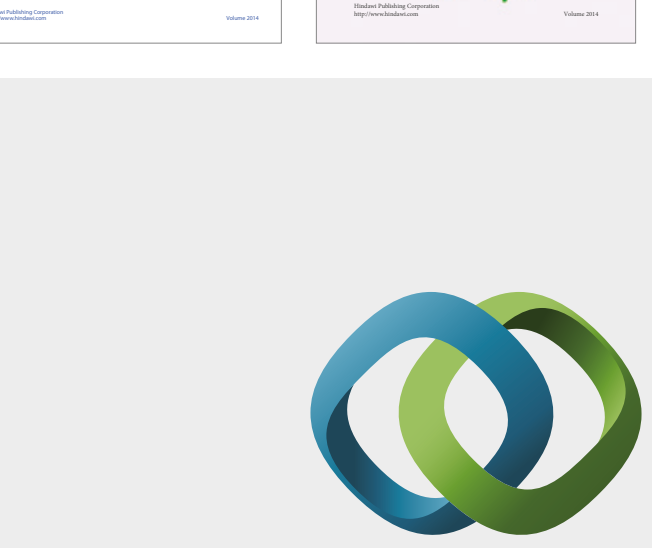

\section{Hindawi}

Submit your manuscripts at

https://www.hindawi.com
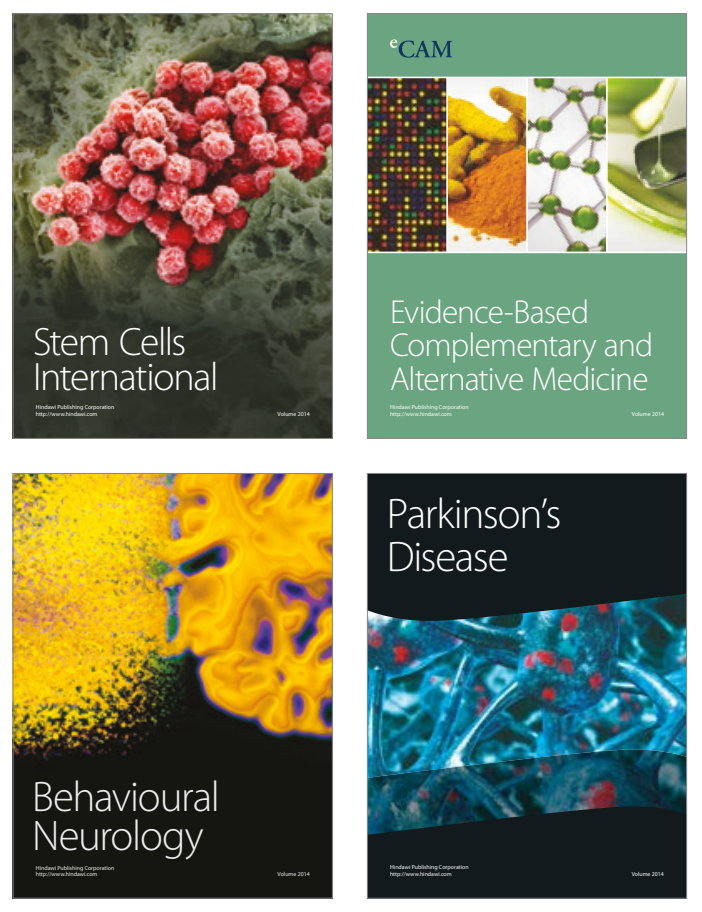
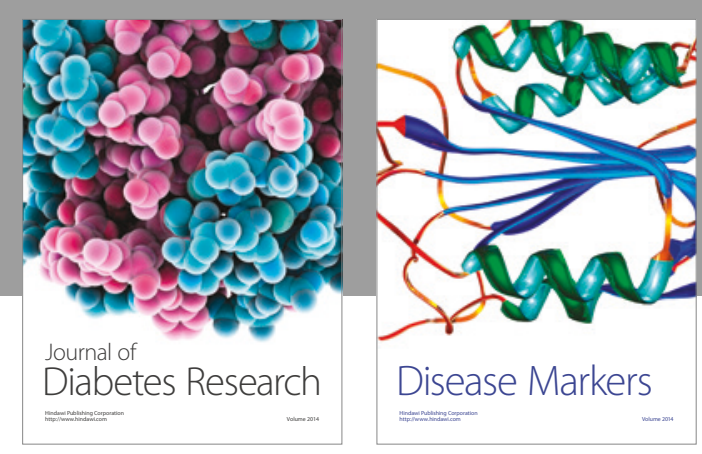

Disease Markers
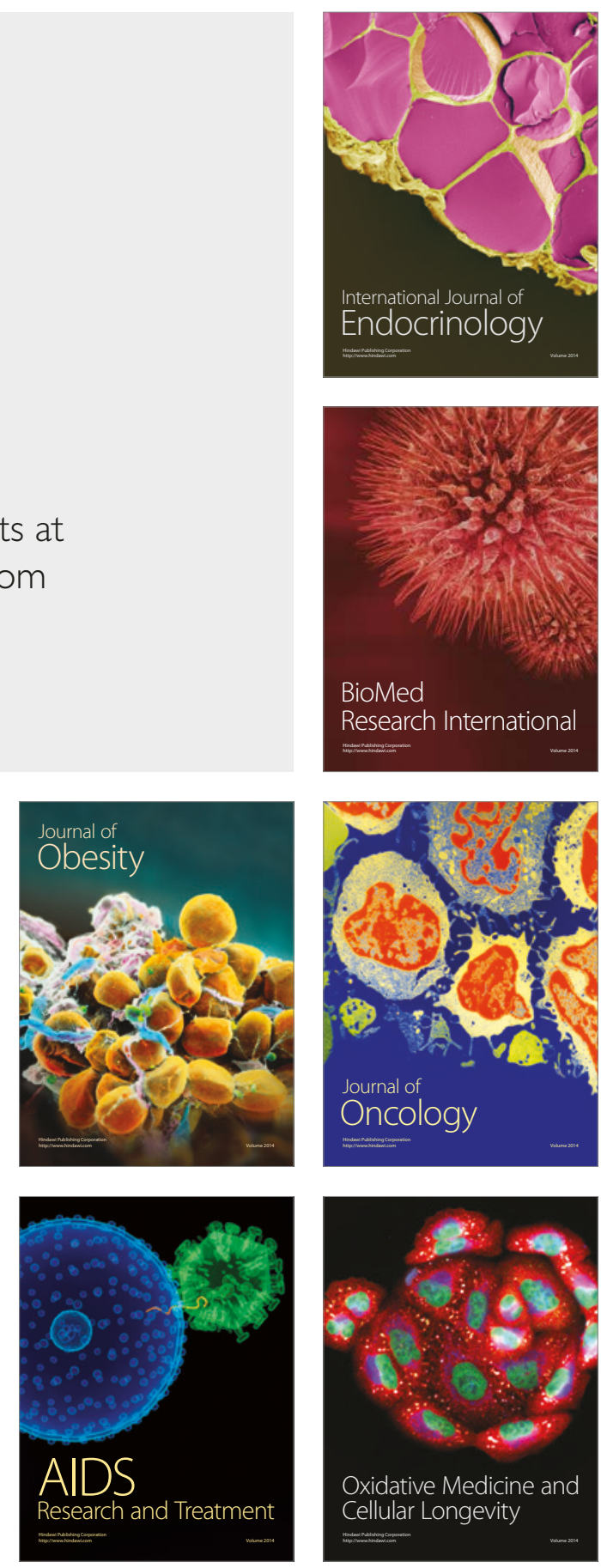